Pierre-Alain Duc, Jonathan Braine and Elias Brinks, eds.

\title{
Tidal Remnants and Intergalactic H II Regions
}

\author{
Tom Oosterloo, Raffaella Morganti
}

Netherlands Foundation for Research in Astronomy, Dwingeloo, The Netherlands

Elaine M. Sadler

School of Physics, University of Sydney, NSW 2006, Australia

Annette Ferguson

Max-Planck Institute für Astrophysik, Karl-Schwarzschild-Str. 1, 85748

Garching, Germany

Thijs van der Hulst

Kapteyn Astronomical Institute, RuG, Landleven 12, 9747 AD, Groningen, The Netherlands

Helmut Jerjen

Research School of Astronomy \& Astrophysics, Australian National University, ACT 2611, Canberra, Australia

\begin{abstract}
.
We report the discovery of two small intergalactic $\mathrm{H}$ II regions in the loose group of galaxies around the field elliptical NGC 1490. The $\mathrm{H}$ II regions are located at least $100 \mathrm{kpc}$ from any optical galaxy but are associated with a number of large $\mathrm{H}$ I clouds that are lying along an arc $500 \mathrm{kpc}$ in length and that have no optical counterpart on the Digital Sky Survey. The sum of the H I masses of the clouds is almost $10^{10} M_{\odot}$ and the largest $\mathrm{H}$ I cloud is about $100 \mathrm{kpc}$ in size. Deep optical imaging reveals a very low surface brightness counterpart to this largest $\mathrm{H}$ I cloud, making this one of the $\mathrm{H}$ I richest optical galaxies known $\left(M_{\mathrm{HI}} / L_{\mathrm{V}} \sim 200\right)$. Spectroscopy of the $\mathrm{H}$ II regions indicates that the abundance in these $\mathrm{H}$ II regions is only slightly sub-solar, excluding a primordial origin of the $\mathrm{H}$ I clouds. The $\mathrm{H}$ I clouds are perhaps remnants resulting from the tidal disruption of a reasonably sized galaxy, probably quite some time ago, by the loose group to which NGC 1490 belongs. Alternatively, they are remnants of the merger that created the field elliptical NGC 1490. The isolated $\mathrm{H}$ II regions show that star formation on a very small scale can occur in intergalactic space in gas drawn from galaxies by tidal interactions. Many such intergalactic small star formation regions may exist near tidally interacting galaxies.
\end{abstract}




\section{Introduction}

Hierarchical accretion and merging of small clumps appears to be a good description of the formation of early-type galaxies. In many early-type galaxies signatures of recent hierarchical assembly can be observed, particularly in field galaxies. Optical fine structure - shells, dust lanes, "X" structures - revealed through un-sharp masking of optical images suggests a recent accretion event. These fine structures are correlated with the presence of an intermediate age (1-2 Gyr) population of stars (e.g. Schweizer et al. 1990). Moreover, comparing detailed stellar population models with high signal-to-noise optical spectra indicates the presence of an intermediate age population in several early-type galaxies (e.g. Trager et al. 2000). A small but significant fraction of early-type galaxies contain neutral hydrogen. The origin of this neutral hydrogen is generally thought to be external and due to recent accretion of companions. Hence the presence of $\mathrm{H}$ I in early-type galaxies is generally thought to fit into the picture of continued accretion of companions and the associated intermittent episodes of star formation in some early-type galaxies.

We are undertaking a systematic $\mathrm{H}$ I survey, using the Australia Telescope Compact Array (ATCA), of all early-type galaxies south of $\delta<-25^{\circ}$ with $V<$ $6000 \mathrm{~km} \mathrm{~s}^{-1}$ (based on HIPASS, the Parkes All Sky H I Survey, see Barnes et al. 2001 and elsewhere in this volume) to obtain an unbiased sample of $\mathrm{H}$ I-rich early-type galaxies. Such a complete sample will allow us to study the effects of $\mathrm{H}$ I on the evolution of early-type galaxies in different environments, and the relation between $\mathrm{H}$ I and other indicators of recent accretion and star formation (see also Sadler et al. 2002). We can also study whether H I always has an external origin in early-type galaxies or whether in some early-type galaxies the $\mathrm{H}$ I may be related to an earlier phase in the evolution of the galaxies.

During this HIPASS follow-up program we discovered two intergalactic H II regions near the field elliptical NGC 1490 that are probably related to merging/accretion within the loose group of galaxies around NGC 1490, or to the merger that created the field elliptical NGC 1490. Although unusual, these regions seem to fit into the generally accepted picture regarding $\mathrm{H} \mathrm{I}$ in early-type galaxies. We discuss these $\mathrm{H}$ II regions in section 2. However, we find some evidence that some early-type galaxies do not fit into the scheme of continued accretion. This we briefly discuss in section 3 .

\section{Intergalactic $\mathrm{H}$ II regions near NGC 1490}

The HIPASS spectrum of NGC 1490 shows a strong detection with the presence of almost $10^{10} M_{\odot}$ of neutral hydrogen. This detection was followed up with deep $\mathrm{H}$ I observations performed with the ATCA to image the $\mathrm{H} \mathrm{I}$ in the region around NGC 1490. Five objects were detected with a total H I mass of $8.9 \times 10^{9}$ $M_{\odot}$ (Figure 1). Only one $\mathrm{H}$ I cloud has an obvious counterpart (cloud 4) on the Digital Sky Survey in the form of a small galaxy. The other H I clouds seem to lie along an arc of more than $500 \mathrm{kpc}$ long that bends around NGC 1490. About half of the total amount of $\mathrm{H} \mathrm{I}$ is found in one cloud of about $100 \mathrm{kpc}$ in size (cloud 1). Fig. 2 shows a position-velocity plot of two $\mathrm{H}$ I clouds, as marked in Fig. 1. The kinematics of the $\mathrm{H}$ I clouds indicates that they are separate entities. 


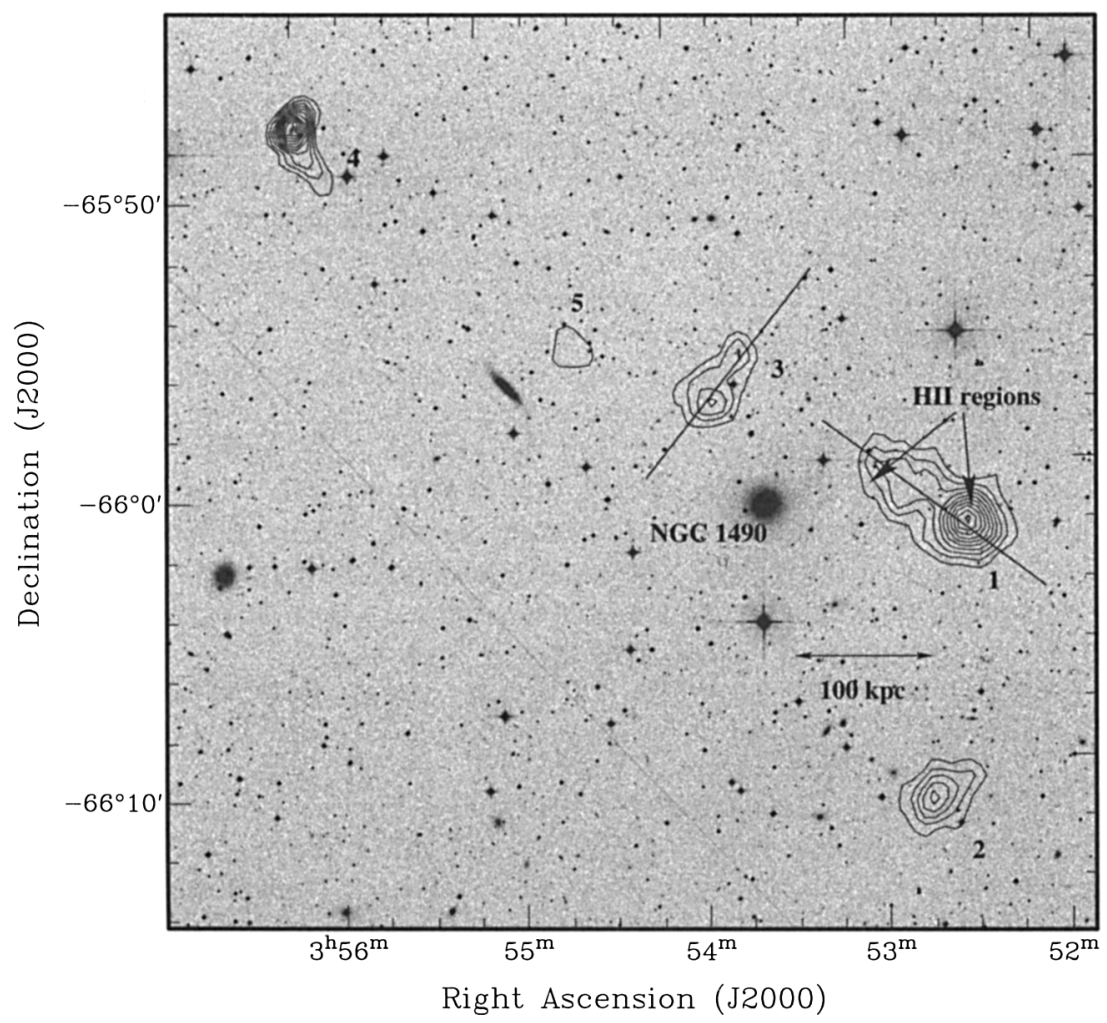

Figure 1. View of the entire region around NGC 1490, displaying the 5 objects detected in $\mathrm{H}$ I. Contour levels: $2,4,6, \ldots \times 10^{19} \mathrm{~cm}^{-2}$. The lines indicate the orientation along which the position-velocity plots of Fig. 2 are taken.

E.g. the velocity gradient in cloud 3 is perpendicular to the line connecting the neighbouring clouds. The "U-shaped" pattern observed in the largest H I cloud indicates that this cloud is not in rotational equilibrium.

Deep optical imaging was performed in a number of standard filters of the region of this largest $\mathrm{H}$ I cloud, using FORS1 on the VLT. This imaging reveals a very low surface brightness optical counterpart (the "OC"). The peak surface brightness in the $\mathrm{V}$ band is $24.5 \mathrm{~V}$ mag $\operatorname{arcsec}^{-2}$. The low surface brightness nature of the $\mathrm{OC}$ is illustrated in Fig. 3. The total magnitude is about 20.5 in $\mathrm{V}$. This implies an extremely high gas-to-light ratio of $M_{\mathrm{HI}} / L_{\mathrm{V}} \sim 200$. For the colour indices we find $\mathrm{U}_{\mathrm{B}}=0.5, \mathrm{~B}_{\mathrm{V}}=0.1 \mathrm{~V}_{\mathrm{R}}=0.4$ and $\mathrm{R}_{\mathrm{I}}=0.6$. These colours indicate the presence of a young stellar population.

We further investigated the nature of the main $\mathrm{H}$ I cloud and its $\mathrm{OC}$ by searching for the presence of $\mathrm{H} \alpha$ emission using narrow-band $\mathrm{H} \alpha$ imaging with the NTT. These images reveal the presence of $\mathrm{H} \alpha$ emission in the OC at the location of the peak of the $\mathrm{H} \mathrm{I}$, indicating that some star formation is occurring in the OC. Most interestingly, two small regions of $\mathrm{H} \alpha$ emission were found at 

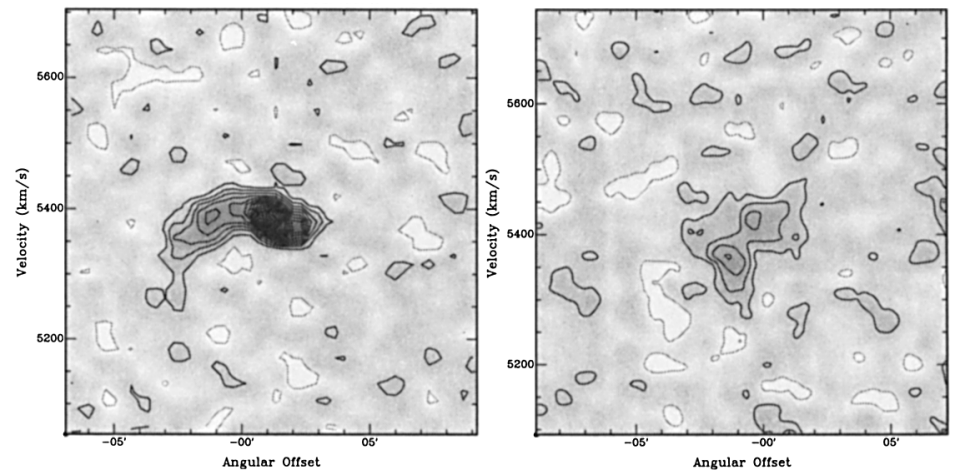

Figure 2. Position-velocity plots of clouds 1 and 3 , taken along the line indicated in figure 1 . Contour levels are $-1,1,2,3, \ldots$ mJy beam $^{-1}$

the edge of the main $\mathrm{H} \mathrm{I}$ cloud, at a distance of about $100 \mathrm{kpc}$ from the $\mathrm{OC}$ (indicated by $1 \& 2$ in Fig. 3). The broad-band images indicate that there is no extended optical continuum light near these two $\mathrm{H} \alpha$ regions.

The $\mathrm{H} \alpha$ luminosities of the $\mathrm{H}$ II complexes are $3-5 \times 10^{38} \mathrm{erg} \mathrm{s}^{-1}$ (i.e. $30-50$ times Orion). The rate of star formation estimated from these luminosities is very modest: around $0.005 \mathrm{Myr}^{-1}$ per region. All the stars corresponding to the diffuse optical counterpart can be formed over a Hubble Time with such a star formation rate.

Optical spectroscopy of the three $\mathrm{H} \alpha$ regions was performed with FORS1 on the VLT. Preliminary analysis of the data suggests that the abundances in the $\mathrm{H} \alpha$ regions are only slightly below solar (in the range $0.5-1.0 Z_{\odot}$ ). This relatively high abundance excludes a primordial nature of the $\mathrm{H}$ I clouds.

\subsection{The nature of the clouds near NGC 1490}

The observations point to a tidal- or merger-related origin for the system of H I clouds near NGC 1490. The alternative hypothesis, i.e. the H I clouds are primordial, can be excluded because of the near solar abundance measured in the $\mathrm{H}$ II complexes. The arc-like arrangement of the $\mathrm{H}$ I clouds is suggestive for a tidal origin. It bears some resemblance to other systems. There is probably a parallel between the objects discussed here and systems like e.g. the Leo Ring (Schneider et al. 1989). Tidal interactions between galaxies and merging is a very diverse process that can produce very spectacular objects with bright tidal tails. But there are also cases where the evidence of an interaction is only detectable in neutral hydrogen. The extreme version is that of a galaxy being completely destroyed by a larger galaxy without any merging taking place. The material of the original galaxy is spread out over such a large volume that, in the optical, the surface brightness is well below the detection limit and only the $\mathrm{H}$ I remains observable (e.g. Ryan-Weber et al. 2003, see also this proceedings). Perhaps the H I clouds near NGC 1490 have such an origin.

An alternative hypothesis is that the $\mathrm{H}$ I clouds are remnants of the merger between two or several gas-rich galaxies that also created the field elliptical 

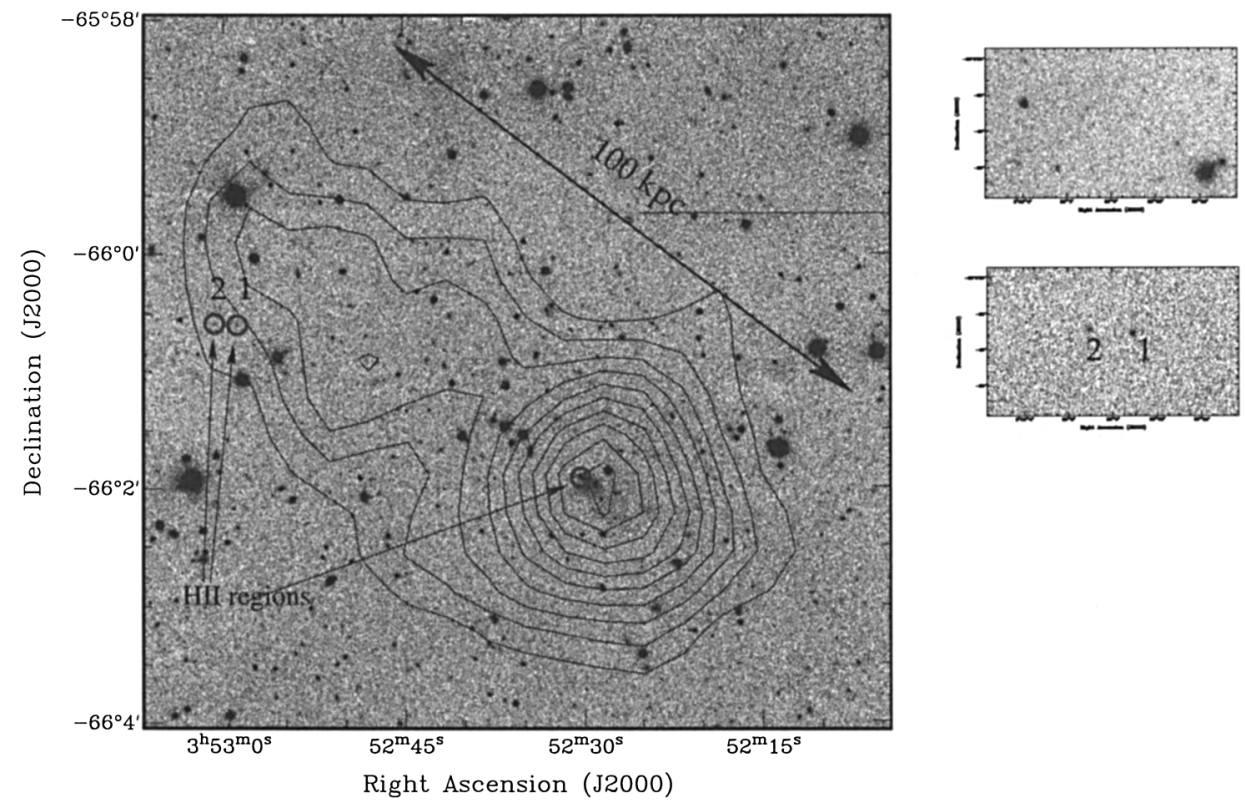

Figure 3. Left $\mathrm{H}$ I contours on top of an $r$-band image obtained with the NTT. Contour levels are as in Fig. 1. The location of the three $\mathrm{H}$ II complex discovered using the narrow-band imaging are indicated. Right Continuum optical image (top) centred on the regions 1 and 2 and the continuum subtracted line image, centred on the redshifted $\mathrm{H} \alpha$, revealing the two $\mathrm{H}$ II complexes (bottom)

NGC 1490. The environment of NGC 1490 can be characterised as a loose group. The presence of an elliptical galaxy in such a group often indicates that merging has occurred in the group.

The interesting aspects of the case presented here are:

1) the very large amount of $\mathrm{H}_{\mathrm{I}}$ involved, distributed in fairly large clouds for which only deep optical imaging reveals an optical counterpart. As the velocity structure indicates, these clouds are separate entities, suggesting they are Tidal Dwarfs even though they have no bright optical counterpart. The clouds are not, or hardly, visible in the optical, but, given the large reservoir of $\mathrm{H} \mathrm{I}$, perhaps at some point in time they will start to make stars and become brighter optical objects.

2) the existence of an extremely gas-rich object with $M_{\mathrm{HI}} / L_{V} \sim 200$. This is perhaps an example of a "Tidal Gas Cloud" that started making stars recently. However, it is perhaps more likely that the optical material originates in a galaxy that was destroyed in an interaction.

3) the presence of small $\mathrm{H}$ II complexes that are located far away from any optically detectable galaxy, in our case more than $100 \mathrm{kpc}$. Only very few examples of such $\mathrm{H}$ II regions are known. The observations presented here suggest that in more systems intergalactic $\mathrm{H}$ II complexes may exist. 

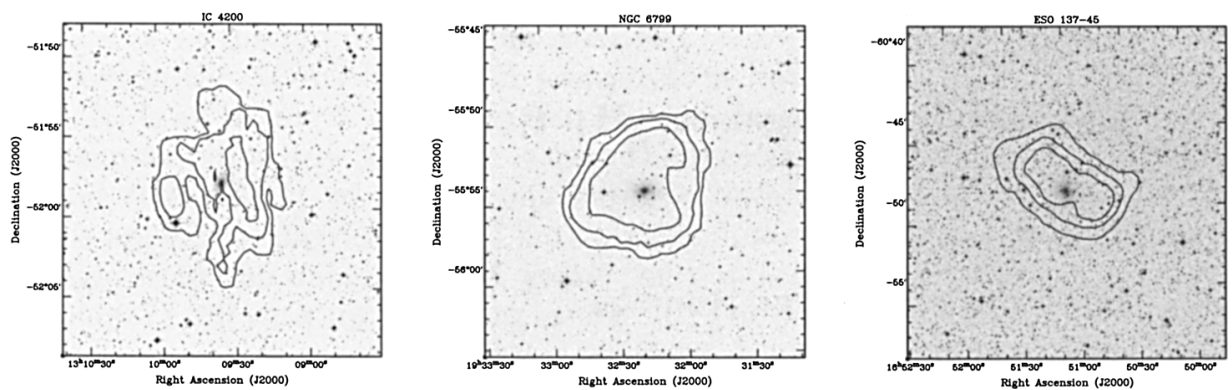

Figure 4. Examples of very large regular disks of $\mathrm{H}$ I distribution around "normal" early-type galaxies obtained from the ATCA follow up of HIPASS detections (see text for details). The disks shown here have sizes between 150 and $200 \mathrm{kpc}$. Contour levels: $2,4,8,16 \times 10^{19}$ $\mathrm{cm}^{-2}$.

\section{Non-accretion origin of $\mathrm{H}$ I in some early-type galaxies?}

As a final remark, we note that, although the results discussed above fit very well into the picture continued accretion by early-type galaxies, and the associated external origin of $\mathrm{H}$ I in early-type galaxies, we have found some indications that some early-type galaxies do not fit into this scheme. Our survey has revealed a surprisingly large number of early-type galaxies that have very large (up to 200 kpc in size), regular disks of low column density H I. In Fig. 4 we present three examples of such large regularly rotating $\mathrm{H}$ I disks. The largest of these disks have $\mathrm{H}$ I masses up to $10^{10} M_{\odot}$. Given their size and regular appearance, these disks must be quite old, in many cases well over $5 \times 10^{9} \mathrm{yr}$. Hence, they are not related to recent accretions and may be related to a much earlier formation phase of these galaxies. Moreover, no large accretion can have occurred by these galaxies, as these would likely have destroyed these large $\mathrm{H}$ I disks, or have triggered significant star formation in the $\mathrm{H}$ I disk. The $\mathrm{H}$ I column density in these disks peaks at only $10^{20}$ atoms $\mathrm{cm}^{-2}$, so, despite the large $\mathrm{H}$ I reservoir, no significant star formation is occurring. These $\mathrm{H}$ I disks will evolve only very slowly and will remain as purely gaseous disks for very long periods of time, provided the environment is not too hostile for these disks.

\section{References}

Barnes, D.G., et al. 2001, MNRAS 322, 486

Ryan-Weber, E., Webster, R., \& Bekki, K. 2003, ASSL Conf. Proc.281, p223

Sadler E.M., Oosterloo T., Morganti R., 2002 in The Dynamics, Structure \& History of Galaxies, Eds. G.S. Da Costa and Helmut Jerjen, ASP Conference Proceedings, Vol. 273, p.215

Schneider, S., et al. 1989, AJ, 97, 666

Schweizer F., Seitzer P., Faber S., Burnstein D., Dalle Ore C.M., Gonzáles J.J. 1990, ApJ 364, L33

Trager S.C., Faber S., Worthey G., Gonzáles J.J. 2000, AJ 120, 165 